\title{
Architecture for Resource Allocation in the Internet of Vehicles for Cooperating Driving System
}

\author{
Nafeesa kalsoom $\mathbb{D}^{1},{ }^{1}$ Iftikhar Ahmad $\left(\mathbb{D},{ }^{1,2}\right.$ Roobaea Alroobaea $\mathbb{D}^{\circ},{ }^{3}$ \\ Muhammad Ahsan Raza, ${ }^{4}$ Samina Khalid $\mathbb{D}^{1},{ }^{1}$ Zaheed Ahmed $\mathbb{D},{ }^{5}$ and Ihsan Ali $\mathbb{D}^{2}$ \\ ${ }^{1}$ Department of CS and IT, Mirpur University of Science and Technology (MUST), Mirpur-10250, AJK, Pakistan \\ ${ }^{2}$ University of Malaya, Faculty of Computer Science and Information Technology, Kuala Lumpur, Malaysia \\ ${ }^{3}$ Department of Computer Science, College of Computers and Information Technology, Taif University, P. O. Box 11099, \\ Taif 21944, Saudi Arabia \\ ${ }^{4}$ Department of Information Technology, Bahauddin Zakariya University, Multan 60000, Pakistan \\ ${ }^{5}$ Faculty of Computing and Engineering, University of Kotli, AJK, Kotli, Pakistan
}

Correspondence should be addressed to Iftikhar Ahmad; ify_ia@yahoo.com and Ihsan Ali; ihsanalichd@siswa.um.edu.my

Received 3 December 2020; Revised 12 January 2021; Accepted 21 January 2021; Published 1 February 2021

Academic Editor: Muhammad Arif

Copyright (c) 2021 Nafeesa kalsoom et al. This is an open access article distributed under the Creative Commons Attribution License, which permits unrestricted use, distribution, and reproduction in any medium, provided the original work is properly cited.

\begin{abstract}
Internet of Vehicles (IoV) is a complex system that consists of resource types such as vehicles, humans, and sensors. Although the Internet of Vehicles is complex, it improvises communication among vehicles on the roads. Quality of service (QoS) enabled the cooperative driving system (CDS) based on 5G technology, enabling vehicles to communicate and cooperate to improve road traffic efficiency. Due to the high vehicle density and limited resources (bandwidth) of current network infrastructure, sometimes a better channel that meets the requirements of cooperative driving is not available that causes network congestion, which directly influences the overall QoS of the CDS. To overcome this, we proposed a $5 \mathrm{G}$ network-based architecture for CDS that incorporates a D2D technology-based resource allocation scheme. The proposed network architecture and cooperative behavior-based scheme helps in improving QoS for CDS. We implemented our proposed scheme by incorporating the density-based scattered clustering algorithm with noise for vehicular clustering. The proposed scheme's performance shows significant improvement in terms of throughput compared with existing D2D approaches.
\end{abstract}

\section{Introduction}

The fifth generation (5G) desires to be a generation that is not extended only to meet the needs of mobile operatives and consumer infrastructures and opens up new paradigms and support for societies, health, vehicles, media, manufacturing, and the transport industry [1]. 5G is seen as the underlying technology allowing self-driving cars to talk with each other. Cooperative driving plays a vital role in traffic efficiency. The new development of information and communication technologies allows auspicious cooperative driving, which has been meditated to enhance traffic efficiency and traffic safety [2] expressively.
Cooperative driving systems (CDS) are primarily based on wireless communication between vehicle-to-vehicle (V2V) and among vehicles to infrastructure (V2I). Human behavior is modelled in CDS that helps vehicles while travelling on roads [2]. The exchange of information among vehicles enables them to make cooperative decisions regarding driving [3].

IoVs prepared with $5 \mathrm{G}$ interfaces are complex but give additional features because of D2D communication. The base station plays a role by directing vehicles with control information to schedule the resources as per priority. In this way, base station guarantees the available resources to meet the latency and data rate required for typical vehicles [4]. 
Resource allocation procedures in most fourth-generation telecommunication networks require continuous monitoring of channels but do not provide required resources to every vehicle as per their needs [5]. Although 5G is quite capable of the service requirement of $\mathrm{V} 2 \mathrm{~V}$, its use must be controlled to make it cost-effective [6]. The main motive is the efficient utilization of the radio resources as per increasing QoS demand for CDS.

High vehicle density that shares a common channel for communication QoS is not filled in CDS. So, clustering for $5 \mathrm{G}$ users is better for efficient resource utilization and allocation. CDS for 5G-based V2V communication is necessary to provide better resources like bandwidth to improve QoS requirements [7]. Due to extreme automobile density and limited bandwidth of current network infrastructure, sometimes a better channel that meets the requirements of cooperative driving is not available that causes network congestion. Simultaneously, the ever-changing topologies of communicating vehicles are a big hurdle while allocating appropriate resources at the right time and place. These things can directly impact the overall QoS performance of the CDS. For better V2V communication to improve service requirements, it is necessary to develop a network infrastructure that improves the QoS of cooperative driving with a greater throughput.

This paper proposed a resource allocation scheme for a cooperative driving system for IoVs. First, an architecture of the cooperative driving system (CDS) is proposed based on $5 \mathrm{G}$ networks. Then, a clustering algorithm is proposed for resource allocation. Consequently, proposed architecture entities, how these entities work, and their functionalities for our proposed solution are outlined. Secondly, a clustering algorithm for IoVs to allocate resources for better transmission in CDS is formulated. The proposed scheme is implemented, and the results are validated against the existing scheme. Section 2 discusses the related work, and Section 3 presents the proposed CDS architecture in detail. Section 4 presents the proposed solution's implementation, and the performance evaluation is presented in Section 5 . Finally, Section 6 presents the conclusion of the paper.

\section{Related Work}

The automation of vehicles is increasing over time. Simultaneously, technological advancement in telecom networks increases the number of interactions among vehicles and between vehicles and other road users. As a result, the communication system's reliability and capability become significant for improved performance of autonomous driving and road safety [8].

Three usual multivehicle cooperative scenarios, formation control, convoy-driving, and connection management are studied in [9]. A communication resource allocation solution was brought forth for these three scenarios. The concentration was on just one convoy when it crosses the intersection. A self-organized resource allocation scheme for $\mathrm{V} 2 \mathrm{~V}$ communication is suggested in [10]. To lessen the overhead dynamic, zone formation-based resource algorithm is proposed. This scenario's problem is that multiple vehicle zones are connected with only one roadside units (RSUs). It causes transmission overhead for the base station and reduces the channel state quality. The problem here is that every V2I links bonds spectrum with multiple V2V associations. The BS has only access to gentle decreasing certificate status information (CSI) of vehicular associations, except those who are dismissing at the BS [11, 12].

A cooperative allocation strategy for bandwidth against each link is presented in [13] to achieve better communication performance among vehicles on the move. CAVDO and MA-DTR-based clustering approaches are used to organize network organization and resource allocation $[14,15]$. It performs fine in clustering by dropping the number of clusters when there is very high vehicle density. However, if clustering is not appropriately done, resource optimization is mislaid.

Höyhtyä et al. [16] analyzed the Third-Generation Partnership Program (3GPP) standards for power consumption management regarding 5G. Release 14 presents two types of innovative communication modes that are specified for $\mathrm{V} 2 \mathrm{~V}$ communications. In mode three, the cellular networks choose and achieve the cars' radio resource for their direct V2V communication. In mode 4, vehicle automatically chooses the radio resources for their direct $\mathrm{V} 2 \mathrm{~V}$ communications. Mode 4 could function without cellular coverage. It has a different scattered scheduling system for vehicles that choose their radio resources and provides functioning for distributed congestion control $[17,18]$.

A comprehensive analysis of 5G [19] related to road traffic management is presented with different problems that occur during the resource allocation. Two main challenges for D2D communication are first interference for D2D links. Secondly, it improves QoS requirements using RRM and RRM scheduling algorithms in the neural Q-learning-based approach [20]. An ACORA [21] scheme (ant colony optimization-based resource allocation algorithm) is proposed, which promises the efficient use of accessible resources helping users by conferring their QoS parameters. A swarm intelligence algorithm is adopted to reduce computational complexity while understanding suitable performance. RRM [22] for V2X based on D2D communication is proposed which promises reliability and latency while allocating resources to vehicles.

A clustering scheme based on cooperation among vehicles is presented in [23], which is dependent on GPS. Performance is a lack in case of GPS signal interruptions. Another LTE-dependent clustering scheme is presented in [24]. It relies on the LTE network for location-based information required by the vehicles while travelling on the road, whenever the need to make driving-related decisions. There is no localized mechanism that helps vehicles in the absence of GPS signals. A Fermi learning model based on the prospect theory is developed in [25], which calculates the distribution density and the distance between the taxis. The authors in [26] proposed an optimal beacon transmission rate allocation for cluster control. An adoptive rate is defined for efficient intracluster communication that is useful in avoiding cluster congestion. 
A 5G network-based control framework [27] is proposed and deployed in Sweden that minimizes communication delay in managing intersections. This framework works cooperatively but no focus on radio resources management. A comprehensive review presented in [28] radio resource management schemes proposed for LTE/LTE-A networks is still relevant. However, these schemes should be adaptive and futuristic that meets the user's demands.

Most of the research done in IoVs for resource allocation that we find in literature used the Knap Sank algorithm [29] and the greedy algorithm. There is a large "network load" during communication that causes "traffic congestion," thus the reason for trouble in large data handling. "Dynamic topology" of both communicating vehicles may reduce network performance [3]. We need such a system that allows vehicles to communicate in dense areas with fewer transmission delays and a better resource allocation system.

\section{CDS Architecture}

A resource allocation architecture and its entities for CDS are proposed in this section, as presented in Figure 1. Symbols and abbreviations used in our proposed design are described in Table 1.

A CDS-based V2V communication helps vehicles to cooperate and communicate with each other on the road to improve traffic efficiency. QoS providence is important for efficient communication. It is necessary to provide proper resources like bandwidth to improve QoS for CDS. To enhance CDS for $\mathrm{V} 2 \mathrm{~V}$ communication, we proposed a clustering algorithm that is DBSCAN for resource allocation in IoVs.

This system's constraints are that devices used in communication should perform in a real-time scenario. If the system does not perform real time, the system working does not handle it. Our system should meet the computational time. The details of the proposed architecture and its algorithm are described below in Sections 3.1 to 3.7.

\subsection{Channel State and Mobility Management Entity. In 5G} networks, the mobility management entity (MME) covers $5 \mathrm{G}$ user location servers connected with the ENodeB access interface. It collects the vehicle's location data. It checks how many vehicles are moving on the road, how many resources are available for each vehicle, and how much additional is needed. The channel state entity, along with the mobility management entity, collects channel state quality information of each ENodeB and exchanges channel state quality information with TES. It updates the information on available resources and the number of resources needed for each vehicle using the banker's algorithm. It checks the resource availability (in our scenario bandwidth), how much resource is needed, how many resources available on each ENodeB, and how much each ENodeB request. The working of channel state entity is as follows:

$$
\begin{gathered}
\text { available }=\text { available }- \text { request }, \\
\text { allocation }=\text { allocation }+ \text { request }, \\
\text { need }=\text { need }- \text { request. }
\end{gathered}
$$

In equation (1), for availability of bandwidth, a resource is subtracted from the requested resources, and then, in (2), an allocated resource is added to the requested resource. To avoid network congestion (3), we apply the formula in which the resource's need is subtracted from the requested resource. The mathematical formulation of equations (1)-(3) is represented in equations (4)-(6) and explained as follows. LTE standards presented a new D2D interface that allows LTE to provide traffic efficiency and a traffic safety communication system. In the system architecture of LTE, the D2D communication interface is called the side link. For resource allocation, a resource pool is created, in which the number of subframes and the number of resource blocks are defined. Let us create a resource pool $R_{P}$ in which the number of subframes is denoted by $S$, and the resource blocks are represented by $R_{B}$, where

$$
\begin{aligned}
S & =\left\{S_{0}, S 1, S 2, \ldots, S_{N-1}\right\}, \\
R p & =\sum_{R p-1}^{R p}+S, \\
R_{B} & =\sum_{R_{B}-R_{P}-1}^{R_{B}}+S .
\end{aligned}
$$

The allocated resource in our scenario is bandwidth represented by $n$. The range of resources is found by a comparison value in which bandwidth is less than the total resource block. For that total resource, blocks are subtracted by 1 and then divided by 2 :

$$
\begin{aligned}
\text { bandwidth } & =n, \\
\text { range } & =n<=\left[\frac{\mathrm{B}_{\mathrm{Rp}-1}}{2}\right] .
\end{aligned}
$$

To allocate resources and to calculate the availability and need of resources, we formulated an equation in which $\log _{2}$ is taken. Resource allocation is represented by $R_{a}$, and resource availability is denoted by $R_{a v}$ :

$$
\begin{aligned}
& R_{a l}=\left[\log _{2}\left(n^{\mathrm{RB}-1}+\frac{1}{2}\right)\right], \\
& R_{a v}=\log _{2}\left[n^{\mathrm{Rp}-\mathrm{RB}}+1\right] .
\end{aligned}
$$

To find a need $(N)$ of resources, available resources are subtracted from allocated resources. So, from equations (8) and (9), we get 


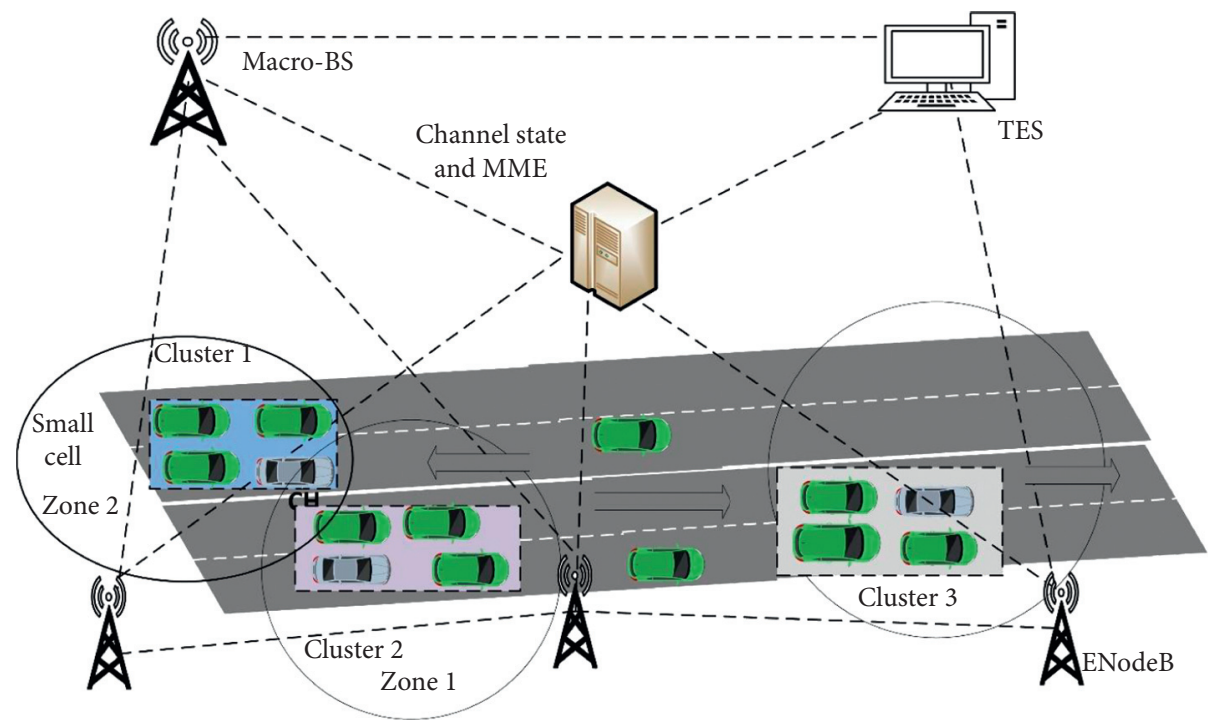

FIGURE 1: Proposed architecture for resource allocation in CDS.

TABLE 1: Symbols used in proposed design and implementation.

\begin{tabular}{lc}
\hline Symbol & Description \\
\hline CDS & Cooperative driving system \\
BOLTZ & Boltzmann constant \\
SNR & Signal to noise ratio \\
BW & Bandwidth \\
$\mathrm{R}_{\mathrm{B}}$ & Resource block \\
$\mathrm{R}_{\mathrm{P}}$ & Resource pool \\
$\mathrm{N}_{\text {R }}$ & Number of resource blocks \\
$\mathrm{N}_{\mathrm{B}} \mathrm{USER}$ & Total number of users \\
$\mathrm{N}_{\text {SC }}$ & Number of subscribers \\
$\mathrm{R}_{\mathrm{a}}$ & Resource allocation \\
$\mathrm{R}_{\mathrm{av}}$ & Resource availability \\
$\mathrm{S}$ & Number of subframes \\
$\mathrm{N}$ & Need of resources \\
$\mathrm{n}$ & One user value \\
\hline
\end{tabular}

$$
N=R_{a v}-R_{a l}\left(N=\log _{2}\left[n^{\mathrm{Rp}-\mathrm{RB}}+1\right]-\left[\log _{2}\left(n^{\mathrm{RB}-1}+\frac{1}{2}\right)\right]\right) .
$$

Controlled and shared resources define the resources in the resource pool. Controlled are the available resources, and the shared resources are the allocated resource. When the resource allocation process is completed, this information is sent to TES for further processing.

3.2. Traffic Efficiency Server (TES). TES receives vehicle location and updates vehicle location messages received from vehicles with a mobility management entity. It stores and updates data exchanged form channel state entity. Based on the channel state information exchanged from the channel state entity, TES allocates resources to the ENodeB for V2V communication [30]. Due to the CDS' strict latency and reliability requirements for $\mathrm{V} 2 \mathrm{~V}$ communication, a traffic efficiency server must manage and accept the network promptly [31, 32], so TES and ENodeB must establish their connection in real time without delay.
3.3. Macrobase Station and ENodeB. Macrobase stations are fundamental elements in any heterogeneous network wireless infrastructure to provide coverage and support capacity. A small cell that is ENodeB is a major infrastructure part of the $5 \mathrm{G}$ network architecture. In our scenario, macro-BS, ENodeB, and TES are interconnected. Resources like bandwidth are assigned to different ENodeBs. In our scenario, the resource is bandwidth. These base stations (ENodeB) handover resources to vehicles. If the requested resource is not available in the base station, it can currently request TES for a particular service. TES server requests a macrobase station. From the macro-BS, these resources are handed over to ENodeB using LTE-A.

3.4. Clustering. Vehicular clustering is a technique of grouping nodes in several clusters. Each cluster has two components: $\mathrm{CH}$ and cluster member (CM) [33]. In V2V communication, CH's role is to lead the group, and all the participants in the group are its members called CM [34]. In our proposed architecture, the closest to ENodeB is the clustering head, and all neighbor nodes are its members. These are active clusters that are involved in $\mathrm{V} 2 \mathrm{~V}$ communication.

3.5. Zone Formation. To cater to interference, each cluster forms its zone for V2V communication [10]. For D2D communication, we set the radius, which is the communication zone. The purpose of creating a zone is that each $\mathrm{CM}$ communicates within its range. When the nodes (vehicles) are in the radius range, the data transmission is high. As soon as they go away from the base station radius, their throughput decreases. Each vehicle communicates in its zone. When a vehicle moves from one zone to another, it makes its new cluster.

A problem arises when many clusters are in communication within their radius. Some intermediate nodes cause interruption because they are connected with both ENodeB. 
A value is set for each zone so that each node communicates within its specific range of ENodeB. It helps to avoid overlapping of the node between the clusters. In Figure 2, the vehicle in the middle of two cluster cause overlapping between the two zones and cause signal interruption for both clusters results in the wastage of the resources during communication.

3.6. Density-Based Spatial Clustering Algorithm for Resource Allocation. In V2V communication, mostly $k$ means clustering technique is used in which the central node is considered as clustering head. We use the DBSCAN algorithm [35] for clustering. It consists of epsilon (Eps) that tells how close the points should be to each other in clusters. Minimum points (Min points) show the minimum number of points to form a dense region. The points which are near the border are included as noise. In our proposed solution, first, we create a resource pool for D2D communication, in which resources are calculated. A subframe is created along with the allotted bandwidth.

For the clustering algorithm, we made a dataset for vehicles. The allotted values in the resource pool are given along with throughput. This is real data gathered from Monte Carlo simulation [36], which is used to get data for D2D communication in Matlab [37]. The clustering algorithm is applied to a dataset that contains labelled data. When the cluster formation is done, the resource allocation process is started. Equation (11) shows the DBSCAN algorithm's working for distance calculation:

$$
\operatorname{dist}(p, q)=\sqrt{\sum_{i=1}^{n}\left(p_{i}-q_{i}\right)^{2}}
$$

3.7. Pseudocode of Proposed Algorithm. For mode selection and transmission point for resource allocation for CDS is given in (Algorithm 1).

\section{Implementation Scenario}

In our implementation, the first resource pool is created, and then, the DBSCAN algorithm is implemented on the dataset. This dataset is created by taking the data from the D2D simulator. D2D throughput is calculated using Monte Carlo simulation. As per our system requirements, we design system architecture, two nodes, two base stations with TES, and MME-based V2V communication. Vehicle clustering using the DBSCAN algorithm is incorporated with system architecture.

We create a resource pool in which subframes and resource blocks are created. To allocate resources, equations (1), (2), and (3) are defined as presented in Section 3.1. Figure 3 illustrates the $\mathrm{V} 2 \mathrm{~V}$ resource pool for our resource allocation. Figure 3 shows that the $\mathrm{V} 2 \mathrm{~V}$-controlled resource pool is dark blue, and the shared resource pool is yellow. The light blue color shows synchronization time. The brown color shows the baseband and the green color for bandwidth. After the simulation is done in Matlab, the results are compared with the D2D communication model [38].

4.1. Implementing the DBSCAN Algorithm. After creating a resource pool, we create a dataset for resource allocation and implement the DBSCAN algorithm for that dataset, as shown in Figure 4. The dataset is used for the clustering of data. It is a well-known clustering algorithm used in machine learning and data mining. It consists of Eps that tells how close the points should be to each other in clusters.

Min points show the minimum number of points to form a dense region. The points which are near the border are included as noise. This is a labelled dataset. Each label shows the functionality of every point.

4.2. Throughput Calculation. Equation (12) shows $N_{-} R_{B}$ is the number of resource blocks and N_USER is the total number of users, where $n$ is used for one user value. Equation (13) is used to calculate the total number of subscribed users (N_SC) per resource blocks:

$$
\begin{aligned}
& \text { n_user_R } R_{B}=\left(\frac{N \_R_{B}}{n}\right), \\
& \text { n_user_SC }=\text { n_user_ } R_{B} * \frac{N \_S C}{N \_R_{B}}, \\
& n \_R_{B} \text { user }=\frac{N \_R_{B}}{N \_U S E R} .
\end{aligned}
$$

Equation (14) calculates throughput, and the first SNR is calculated. In the following equation, BW is the bandwidth, and BOLTZ is a Boltzmann constant that is (1.3806488e-23). It is used when we calculate SNR with bandwidth:

$$
\mathrm{SNR}=\left([\text { interference array }]+\mathrm{BOLTZ} * \frac{\mathrm{BW}}{N_{-} R_{B}}\right) .
$$

To calculate the D2D throughput, sum of the $\log$ is multiplied with the SNR value and then add one in it, as shown in the following equation:

$$
\text { D2D_throught }=\operatorname{sum}\left(\log _{2}(1+\text { D2D_SNR })\right) \text {. }
$$

\section{Performance Evaluation}

We measure the performance of the proposed CDS against the D2D communication [38] scheme for vehicular networks. Throughput is measured against the number of RBs, various radius sizes, and the number of users. In the end, SNR against throughput is also presented for performance check. The parameter setting for the calculation of throughput is shown in Table 2 .

Figure 5 shows the average throughput against the allocated number of radio RB. At the lower number of $\mathrm{RBs}$, there is not much difference in the throughput of the existing D2D scheme and proposed scheme. However, this difference increases with the increase in the RBs at $\mathrm{RB}$ of 


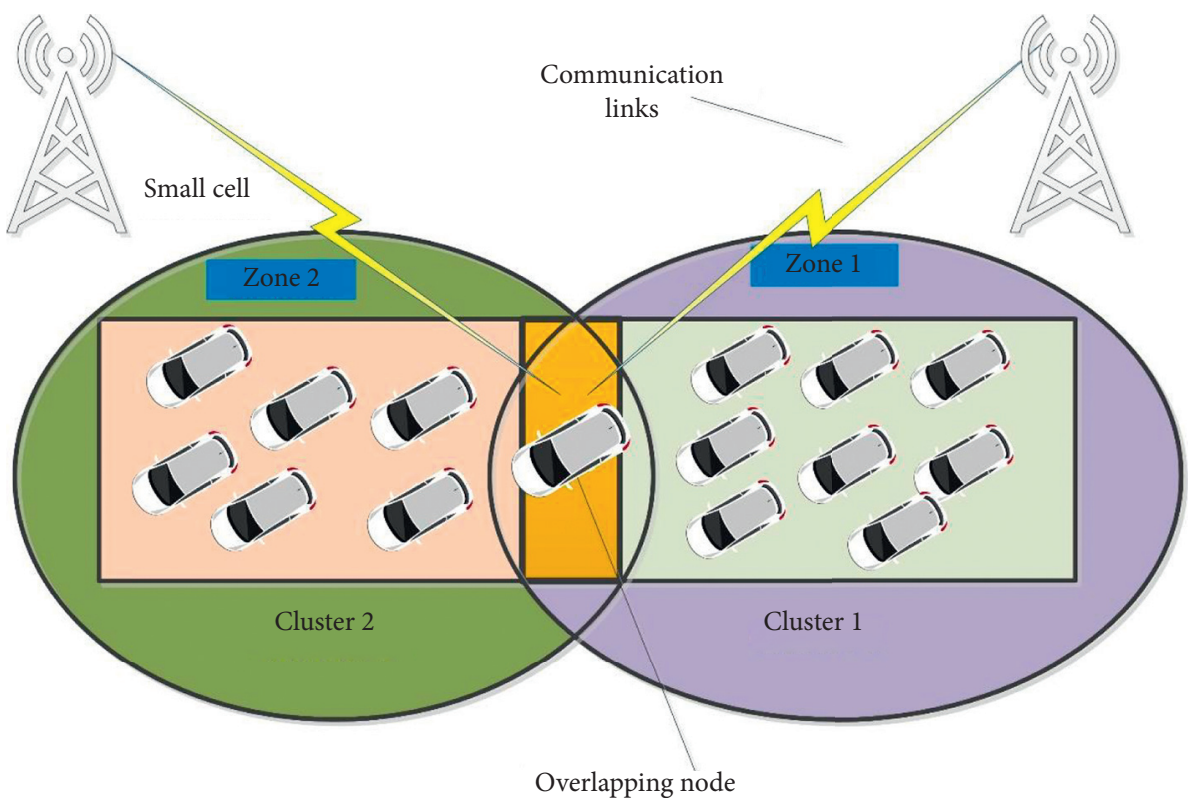

FIGURE 2: Overlapping vehicles cause interruption between vehicles.

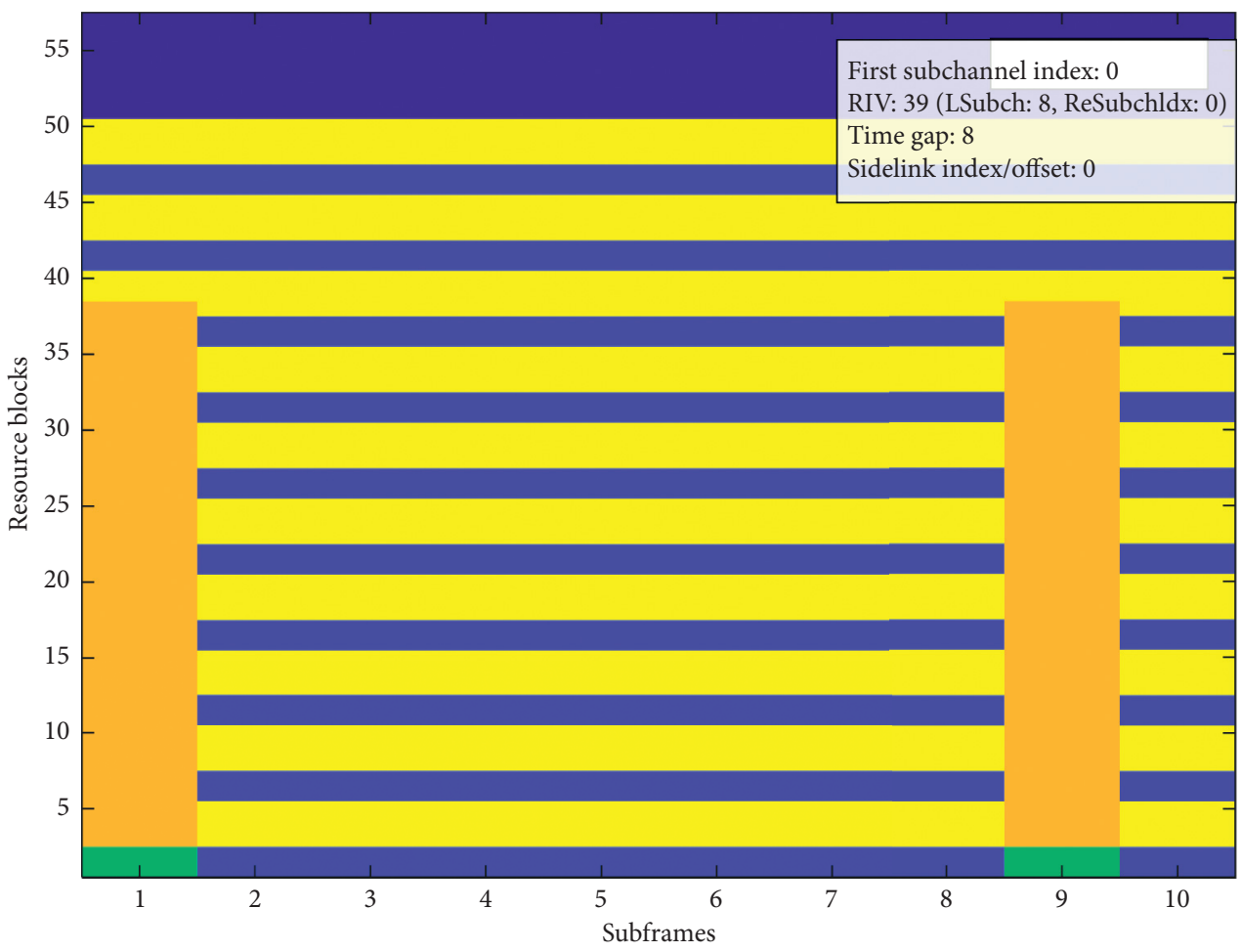

FiguRE 3: V2V resource pool for resource allocation.

100. The throughput of the proposed scheme is almost double the existing D2D schemes. One of the reasons is that, in the D2D scheme, an increase in the network's communication area involves more latency or packet drop.

Average throughput is calculated to find the QoS for $\mathrm{V} 2 \mathrm{~V}$ communication. For the D2D communication scheme, average throughput is calculated for several resource blocks, for the radius size, and the number of users. From the results, it is shown that our proposed algorithm has better results as compared to the existing D2D scheme. The average throughput increases with an increase in the RBs. The increase in the proposed scheme's throughput is higher than the existing D2D schemes. This is because increase in RBs increases the chances for the mobile node to get more required resources on time. 
(1) Initialize transmission point setting for $\mathrm{RB}$ and $\mathrm{PDSSCH}$

(2) FOR each CSI set resource configuration and transport block size

(3) CREATE subframes for Resources allocation

(4) For Each D2D Transmitter d

(5) IF Orthogonal resource block $x$ is available

(6) IF link gain in D2D mode is higher DO

(7) D2D Transmitter $d$ transmits in D2D mode on RB $\mathrm{x}$

(8) End

(9) D2D Transmitter $d$ transmits in UE mode on RB x

(10) ELSE

(11) Update the counter $\mathrm{x}$

(12) END

(13) FOR calculate throughput DO

(14) Allocate resources and decide the mode for D2D transmitter $d$ according to the DBSCAN algorithm where Min point and Eps are defined to $x$.

(15) Update the counter $\mathrm{x}$

(16) END

(17) END

Algorithm 1: For mode selection and transmission point for resource allocation for CDS.

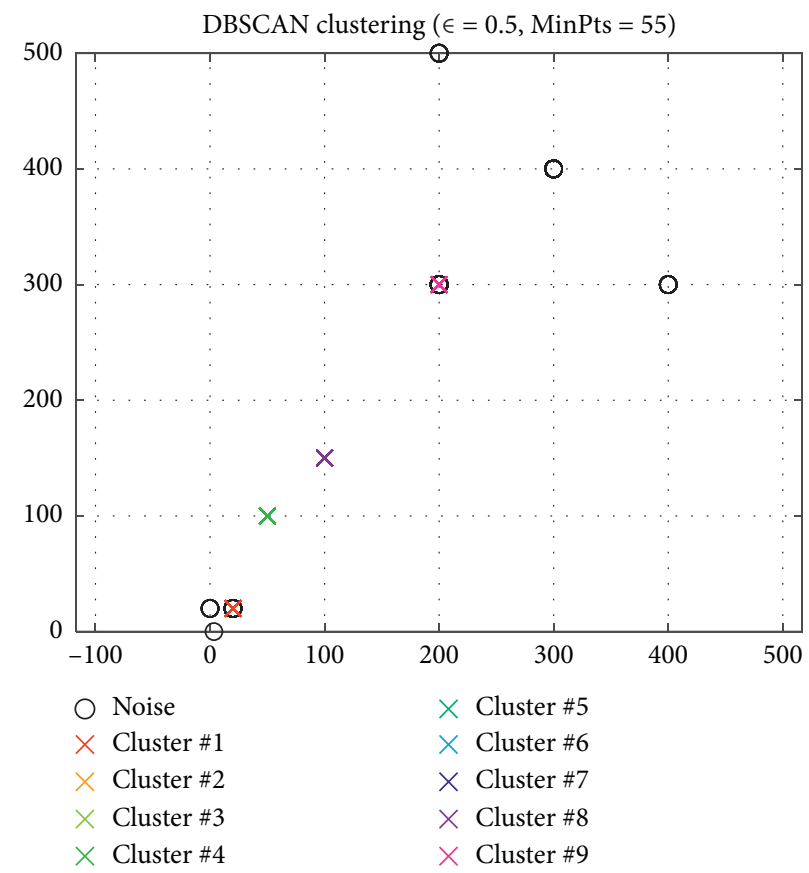

FIGURE 4: Implementation of the DBSCAN algorithm on our dataset.

Figure 6 shows an average throughput against various network radius sizes for both the D2D scheme and the proposed scheme. The average throughput decreases as the radius size is increasing. An increase in the signal rang increases the cluster size as more vehicles are added, affecting overall communication among vehicles. By increasing radius and size, there is a negative impact on both protocols' performance. However, our proposed protocol's performance is still greater at each of the radius size values. The decrease in the performance is because of the increase in transmission failures and an end-to-end delay. This is also evident in Figure 7, which shows an increase in the number of users decreases the throughput as more radius size allows more vehicles to become part of the communication. Thus, 
TABLe 2: Parameters set to calculate throughput.

\begin{tabular}{lc}
\hline Parameters & Values \\
\hline Frequency & $5(\% \mathrm{GHz})$ \\
No. of resources (n_rb) & $20,40,60,80,100$ \\
N_user & 25 \\
Path loss parameter & 3.5 \\
Radius & $25 \mathrm{~m}$ \\
No. of antennas & 2 \\
Transmission mode & FDD \\
No. of the base station & 2 \\
CSI & On \\
\hline
\end{tabular}

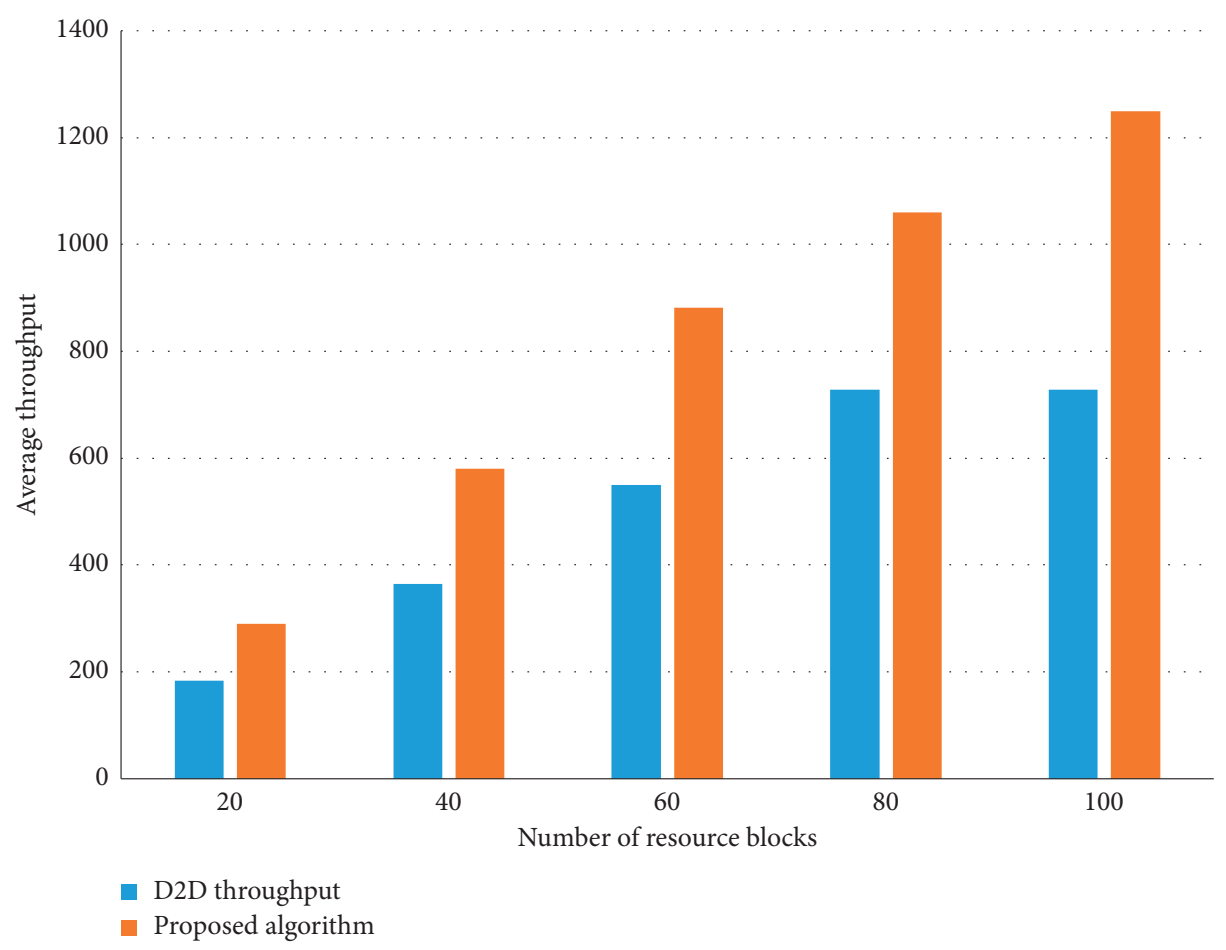

FIgURE 5: Average throughput against allocated no. of RBs.

as the network's scalability is concerned, the proposed scheme has shown better performance. The average throughput remains almost double of the D2D scheme at radius sizes of 30,40 , and 50 meters.

Figure 7 shows the average throughput against the number of users. Our proposed algorithm's average throughput is much higher than a simple D2D-based communication scheme. For the D2D communication scheme, when the number of users increases, the average throughput decreases because all users want to get all resources. In our case, the throughput is good, which provides better QoS for CDS.

SNR directly affects the performance of throughput. When SNR's value is high, it means that the signal's strength is also more robust relative to the noise levels; it allows higher data rates and fewer retransmissions. The lower the SNR value, the lower is the data rate, which decreases throughput.

Figure 8 shows the SNR concerning the throughput of our proposed schemes. In our scenario, the average SNR is high, which increases throughput; thus, the QoS for the CDS increases, which helps traffic efficiency applications. The increase in throughput against various metrics shows that our proposed architecture for resource allocation for CDS performed well against the existing D2D scheme. Throughput increases because of the efficient resource allocation that positively impacts data rate and reduces overall transmission delay. 


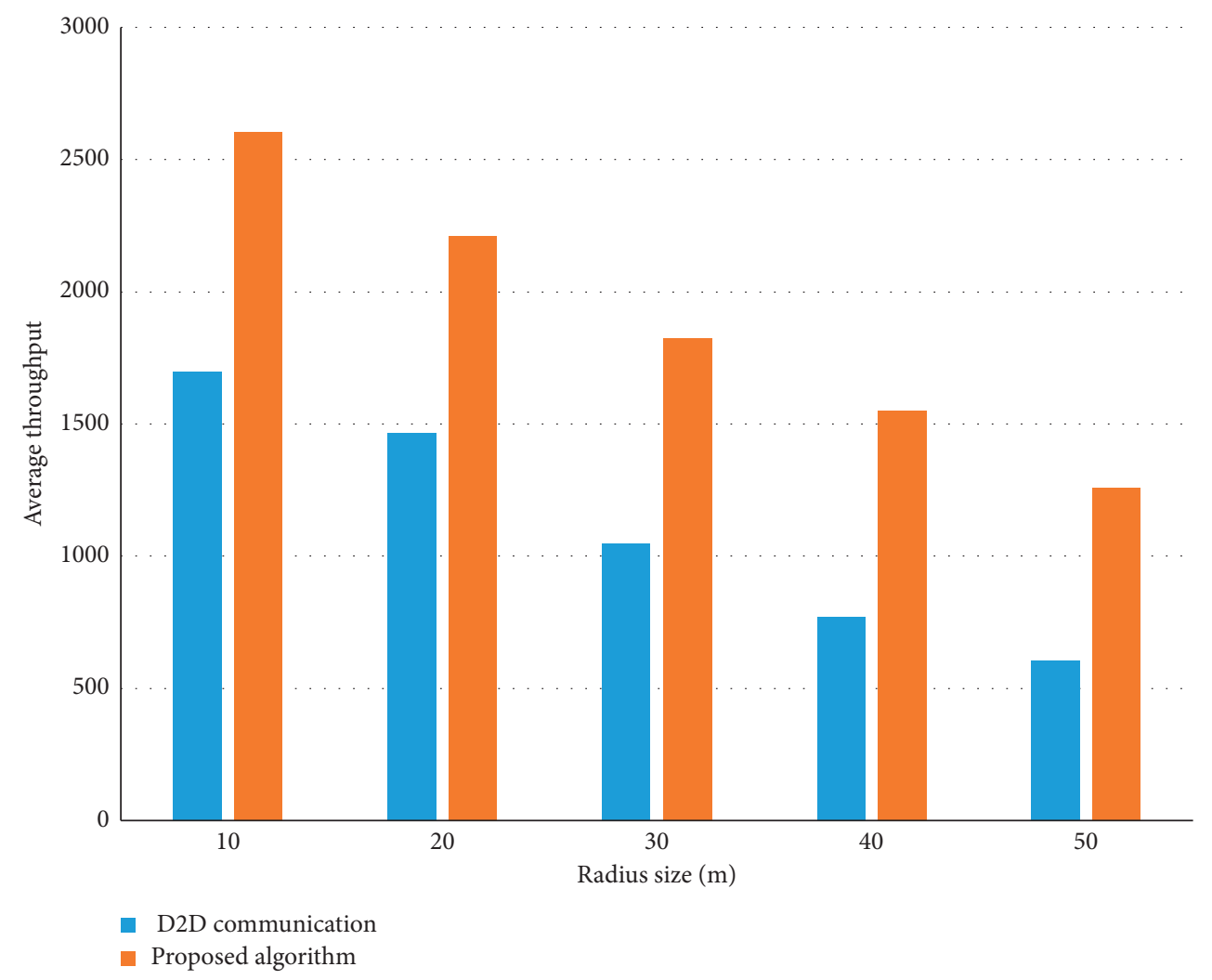

FIGURE 6: Average throughput against various radius size.

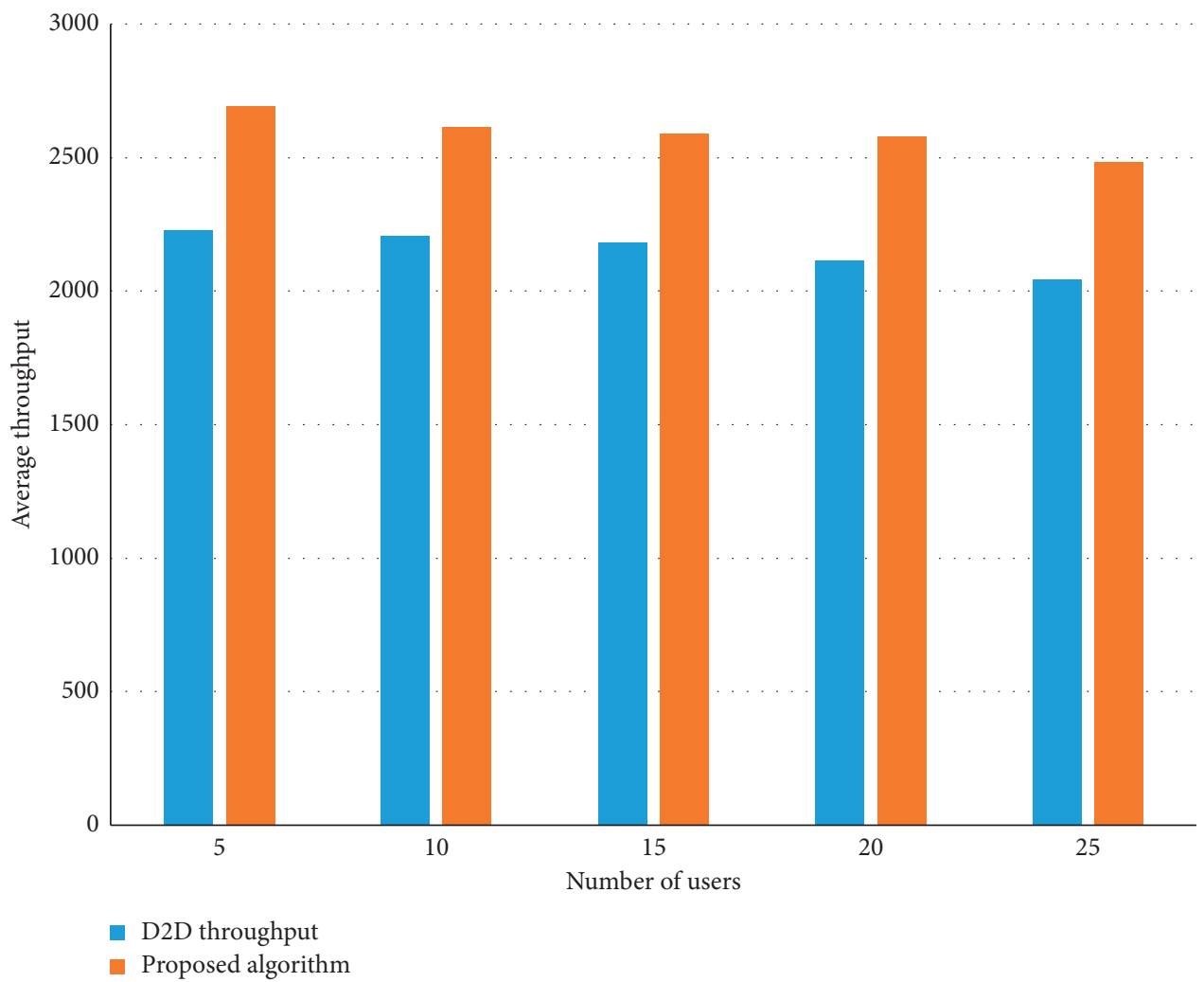

Figure 7: Average throughput against the number of users. 


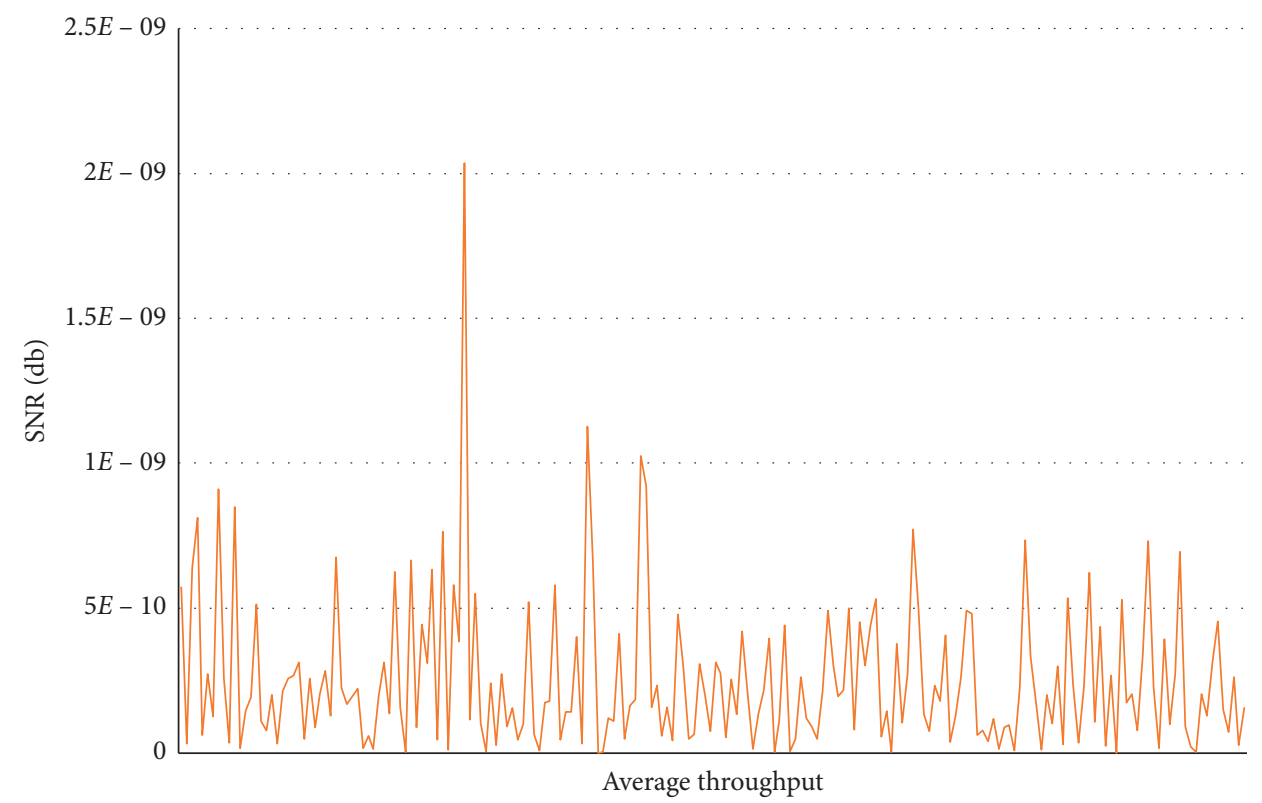

FIgURE 8: Average SNR concerning throughput.

\section{Conclusion}

IoVs is a trend of the IoTs, used for communication among vehicles. Better V2V communication that meets the QoS requirements is essential for efficient road traffic management. A QoS CDS based on V2V communication helps vehicles communicate and cooperate reasonably to improve traffic efficiency over the road as there is a significant increase of more than 50 percent (on average) in the throughput of the proposed CDS than the existing D2D schemes. The results adequately appropriate the main motive of the efficient use of radio resources to meet CDS's QoS requirements. This technique may help in the future to improve QoS for CDS by better allocation of resources.

\section{Data Availability}

The data used to support the findings of this study are available from the corresponding author upon request.

\section{Conflicts of Interest}

The authors declare that they have no conflicts of interest.

\section{Acknowledgments}

The authors are grateful to the Taif University Researchers Supporting Project (number TURSP-2020/36), Taif University, Taif, Saudi Arabia. This research work was also partially supported by the Faculty of Computer Science and Information Technology, University of Malaya, under Postgraduate Research Grant PG035-2016A.

\section{References}

[1] D. K. Sah, D. Praveen Kumar, C. Shivalingagowda, and P. V. Y. Jayasree, "5G applications and architectures," in $5 G$
Enabled Secure Wireless Networks, pp. 45-68, Springer, Berlin, Germany, 2019.

[2] B. Liu, D. Jia, K. Lu, D. Ngoduy, J. Wang, and L. Wu, "A joint control-communication design for reliable vehicle platooning in hybrid traffic," IEEE Transactions on Vehicular Technology, vol. 66, no. 10, pp. 9394-9409, 2017.

[3] D. Jia and D. Ngoduy, "Enhanced cooperative car-following traffic model with the combination of V2V and V2I communication," Transportation Research Part B: Methodological, vol. 90, pp. 172-191, 2016.

[4] H. Seo, K.-D. Lee, S. Yasukawa, Y. Peng, and P. Sartori, "LTE evolution for vehicle-to-everything services," IEEE Communications Magazine, vol. 54, no. 6, pp. 22-28, 2016.

[5] M. Botsov, M. Klügel, W. Kellerer, and P. Fertl, "Location dependent resource allocation for mobile device-to-device communications," in Proceedings of the Wireless Communications and Networking Conference (WCNC), Istanbul, Turkey, April 2014.

[6] C. Yang, J. Li, M. Guizani, A. Anpalagan, and M. Elkashlan, "Advanced spectrum sharing in $5 \mathrm{G}$ cognitive heterogeneous networks," IEEE Wireless Communications, vol. 23, no. 2, pp. 94-101, 2016.

[7] I. De la Iglesia, U. Hernandez-Jayo, E. Osaba, and R. Carballedo, "Smart bandwidth assignation in an underlay cellular network for internet of vehicles," Sensors, vol. 17, no. 10, p. 2217, 2017.

[8] M. Fallgren, R. Vilalta, M. Dillinger et al., "Fifth-generation technologies for the connected car: capable systems for vehicle-to-anything communications," IEEE Vehicular Technology Magazine, vol. 13, no. 3, pp. 28-38, 2018.

[9] H. Liu, H. Yang, K. Zheng, and L. Lei, "Resource allocation schemes in multi-vehicle cooperation systems," Journal of Communications and Information Networks, vol. 2, no. 2, pp. 113-125, 2017.

[10] M. I. Ashraf, M. Bennis, C. Perfecto, and W. Saad, "Dynamic proximity-aware resource allocation in vehicle-to-vehicle (V2V) communications," in Proceedings of the Globecom Workshops (GC Wkshps), Washington, DC, USA, December 2016. 
[11] F. Ahmad, F. Kurugollu, A. Adnane, R. Hussain, and F. Hussain, "MARINE: man-in-the-middle attack resistant trust model IN connEcted vehicles," IEEE Internet of Things Journal, vol. 7, no. 4, pp. 3310-3322, 2020.

[12] F. Ahmad, A. Adnane, C. Abdelaziz Kerrache, V. N. L. Franqueira, and F. Kurugollu, "Trust management in vehicular ad-hoc networks and internet-of-vehicles: current trends and future research directions," in Global Advancements in Connected and Intelligent Mobility: Emerging Research and Opportunities, pp. 135-165, IGI Global, Derby, UK, 2020.

[13] X. Jiang, K. Li, H. Jiang, N. Zhu, and X. Tong, "A bandwidthlink resources cooperative allocation strategy of data communication in intelligent transportation systems," China Communications, vol. 16, no. 4, pp. 234-249, 2019.

[14] F. Aadil, W. Ahsan, Z. U. Rehman et al., "Clustering algorithm for internet of vehicles (IoV) based on dragonfly optimizer (CAVDO)," The Journal of Supercomputing, vol. 74, pp. 1-26, 2018.

[15] A. Zaguia, D. Ameyed, and D. Guessoum, "Mobility prediction in pervasive context-awareness system," International Journal of Applied Engineering Research, vol. 13, no. 9, pp. 6594-6607, 2018.

[16] M. Höyhtyä, O. Apilo, and M. Lasanen, "Review of latest advances in 3GPP standardization: D2D communication in 5G systems and its energy consumption models," Future Internet, vol. 10, no. 1, p. 3, 2018.

[17] V. Vukadinovic, K. Bakowski, P. Marsch et al., “3GPP C-V2X and IEEE 802.11p for vehicle-to-vehicle communications in highway platooning scenarios," Ad Hoc Networks, vol. 74, pp. 17-29, 2018.

[18] A. Zaguia and R. Alroobaea, "Ontological model to predict user mobility," International Journal of Advanced Computer Science and Applications, vol. 10, 2019.

[19] N. Kalsoom and S. Khalid, "Utilization of $5 \mathrm{G}$ in IoVs for road traffic management: a survey," International Journal of Innovative Science and Research Technology, vol. 3, 2018.

[20] F. Souhir, A. Belghith, and F. Zarai, "A reinforcement learning-based radio resource management algorithm for D2D-based V2V communication," in Proceedings of the 2019 15th International Wireless Communications \& Mobile Computing Conference (IWCMC), Tangier, Morocco, June 2019.

[21] S. Feki, A. Belghith, and F. Zarai, "Ant colony optimizationbased resource allocation and resource sharing scheme for $\mathrm{V} 2 \mathrm{~V}$ communication," Journal of Information Science \& Engineering, vol. 35, no. 3, 2019.

[22] A. Masmoudi, S. Feki, K. Mnif, and F. Zarai, "Efficient scheduling and resource allocation for D2D-based LTE-V2X communications," in Proceedings of the 2019 15th International Wireless Communications \& Mobile Computing Conference (IWCMC), Tangier, Morocco, June 2019.

[23] I. Ahmad, R. M. Noor, M. R. Zaba, M. A. Qureshi, M. Imran, and M. Shoaib, "A cooperative heterogeneous vehicular clustering mechanism for road traffic management," International Journal of Parallel Programming, vol. 48, no. 5, pp. 870-889, 2020.

[24] I. Ahmad, R. Md Noor, and M. Reza Z’aba, "LTE efficiency when used in traffic information systems: a stable interest aware clustering," International Journal of Communication Systems, vol. 32, no. 2, Article ID e3853, 2019.

[25] W. Zhang and Y. Fan, "Spatiotemporal characteristics and self-organization of urban taxi dispatch," Complexity, vol. 2020, Article ID 3659315, 11 pages, 2020.
[26] L. Hu, Y. Xiao, and Z. Dai, "Beacon transmission rate allocation optimization under synchronized P-persistent repetition MAC protocol for platooning," Wireless Communications and Mobile Computing, vol. 2020, Article ID 8887134, 18 pages, 2020.

[27] L. M. Castiglione, P. Falcone, A. Petrillo, S. P. Romano, and S. Santini, "Cooperative intersection crossing over 5G," IEEE/ ACM Transactions on Networking, pp. 1-15, 2020.

[28] T. Akhtar, C. Tselios, and I. Politis, "Radio resource management: approaches and implementations from $4 \mathrm{G}$ to $5 \mathrm{G}$ and beyond," Wireless Networks, vol. 27, pp. 1-42, 2020.

[29] H. C. Song and J. Kim, "2018) A study of conceptual connected communication vehicular network using distributed cluster algorithm," in IT Convergence and Security, K. Kim, H. Kim, and N. Baek, Eds., Vol. 450, Lecture Notes in Electrical Engineering, Springer, Singapore, 2017.

[30] A. Paul, A. Daniel, A. Ahmad, and S. Rho, "Cooperative cognitive intelligence for internet of vehicles," IEEE Systems Journal, vol. 11, no. 3, pp. 1249-1258, 2017.

[31] J. Lianghai, M. Liu, A. Weinand, and H. D. Schotten, "Direct vehicle-to-vehicle communication with infrastructure assistance in 5G network," in Proceedings of the 2017 16th Annual Mediterranean Ad Hoc Networking Workshop (Med-Hoc-Net), Budva, Montenegro, June 2017.

[32] W. A. Alosaimi, A. Binmahfoudh, R. Alroobaea, and A. Zaguia, "Location prediction in a smart environment," International Journal of Advanced Computer Science and Applications, vol. 10, 2019.

[33] H.-C. Song and J. Kim, "A study of conceptual connected communication vehicular network using distributed cluster Algorithm," IT Convergence and Security 2017, pp. 11-18, 2018.

[34] I. Ahmad, R. M. Noor, I. Ahmedy et al., "VANET-LTE based heterogeneous vehicular clustering for driving assistance and route planning applications," Computer Networks, vol. 145, pp. 128-140, 2018.

[35] H. Bäcklund, A. Hedblom, and N. Neijman, "A density-based spatial clustering of application with noise," Data Mining, vol. TNM033, pp. 11-30, 2011.

[36] S. Mordechai, Applications of Monte Carlo Method in Science and Engineering, INTECH Open Access Publisher, London, UK, 2011.

[37] K. G. Lim, M. K. Tan, R. K. Y. Chin et al., “Traffic vehicular network modelling for VANET inter-vehicle data scavenging," in Proceedings of the 2018 IEEE International Conference on Artificial Intelligence in Engineering and Technology (IICAIET), Kota Kinabalu, Malaysia, November 2018.

[38] X. Li, Y. Sun, L. Zhou, A. Qi, and S. Zhou, "A resource allocation scheme based on immune algorithm for d2d-based vehicular communication networks," IEEE Access, vol. 7, pp. 122536-122543, 2019. 